\title{
Effects of an Alpha7 Nicotinic Receptor Agonist and Stress on Spatial Memory in an Animal Model of Alzheimer's Disease
}

\author{
Paloma Vicens, ${ }^{1,2,3}$ Diana Ribes, ${ }^{1,2,3}$ Luis Heredia, ${ }^{1,2,3}$ \\ Margarita Torrente, ${ }^{1,2,3}$ and José L. Domingo ${ }^{3}$ \\ ${ }^{1}$ Department of Psychology, Universitat Rovira i Virgili, 43007 Tarragona, Spain \\ ${ }^{2}$ Research Center in Behavioral Assessment (CRAMC), Universitat Rovira i Virgili, 43007 Tarragona, Spain \\ ${ }^{3}$ Laboratory of Toxicology and Environmental Health, IISPV, Universitat Rovira i Virgili, 43201 Reus, Spain \\ Correspondence should be addressed to Paloma Vicens; paloma.vicens@urv.cat
}

Received 23 April 2013; Revised 24 July 2013; Accepted 25 July 2013

Academic Editor: Thomas Van Groen

Copyright (C) 2013 Paloma Vicens et al. This is an open access article distributed under the Creative Commons Attribution License, which permits unrestricted use, distribution, and reproduction in any medium, provided the original work is properly cited.

\begin{abstract}
The aim of the present study was to test the effects of PNU-282987 on spatial learning and memory and hippocampal neurogenesis in both intact and chronically stressed transgenic mice. Transgenic mice with susceptibility to Alzheimer's disease (AD) under immobilization stress and not-stressed animals receiving 0 and $1 \mathrm{mg} / \mathrm{kg}$ of PNU-282987 (PNU) were evaluated in a water maze task. The effects of PNU and stress on proliferation of new cells in the hippocampus of these animals were also assessed. The latency to escape the platform was significantly higher in transgenic stressed mice compared to those in the wild stressed group, as well as in transgenic animals without PNU compared to control wild group. On retention of the task, differences emerged on stressed wild animals, PNU wild group, and stressed wild mice receiving PNU. However, no significant differences were detected on new cell proliferation. The results of the present study did not show any impact of stress in acquisition of a spatial task both in wild and transgenic mice. No clear effects of PNU on acquisition of a spatial task in transgenic mice with susceptibility to AD were detected. Although PNU and stress effects were detected on retention of the task in wild animals, no changes were noted in transgenic mice.
\end{abstract}

\section{Introduction}

Alzheimer's Disease (AD) is characterized by a progressive loss of learning and memory processes and alterations in spatial abilities, confusion, and disorientation. One of the most known hypotheses about the etiology of $\mathrm{AD}$ suggests that neurodegeneration begins with an abnormal processing of amyloid precursor protein (APP), resulting in production, aggregation, and deposition of the peptide $A \beta$, thus facilitating the formation of senile plaques and neuronal death [1]. Among genetic factors, in familiar AD cases, 3 autosomal dominant genes have been identified. Specifically, these encode the precursor of $\mathrm{A} \beta$ protein, presenilin 1 , and presenilin 2, being located on chromosomes 21,14 , and 1 , respectively $[2,3]$. These forms are, however, less abundant. It is expected that other genetic and/or environmental factors also contribute to the development of AD.
Nowadays, multiple strains of transgenic mice with mutations of the peptide amyloid precursor protein (APP) have been developed. They have shown to be very useful in studying this neurodegenerative disorder. Specifically, the transgenic strain B6C3-Tg (APPswe, PSEN1De9) $85 \mathrm{~dB} / \mathrm{J}$ is a double transgenic model expressing a chimera of the amyloid precursor protein (Mo/HuAPP695swe) and a mutation of the human presenilin 1 (of 9-PS1). Both mutations are associated with early onset AD. The "humanized" transgene $\mathrm{Mo} / \mathrm{HuAPP} 695$ swe allows mice to secrete a human $\mathrm{A} \beta$ peptide. These animals develop $\beta$-amyloid deposits in the brain at 6-7 months of age, showing a cognitive impairment at 12 months $[4,5]$. Moreover, they also exhibit impairment in different memory related behavioral tasks [6].

Among environmental factors that may contribute to $\mathrm{AD}$, stress is one of the most important. Stress is currently an unavoidable condition in our daily experiences, 
activating physiological systems and disregulating the body homeostasis [7, 8]. Furthermore, stress is also considered a risk factor for a number of diseases, potentially altering neural functions that impair cognitive processes such as learning and memory through the effects of glucocorticoids levels or stress hormones (cortisol and norepinephrine) in hippocampus. Recently, it has been shown that chronic stress by immobilization accelerates cognitive decline and increases extracellular deposits of $\mathrm{A} \beta$ protein in a transgenic model of $\mathrm{AD}$ [9]. Another recent study has shown that hippocampal cholinergic neurons in male Wistar rats become hypersensitive after chronic exposure to stress [10].

Cholinergic neurodegeneration is considered a significant mechanism of cognitive deterioration in elderly and AD patients [11]. Agents enhancing nicotinic cholinergic transmission have been identified as promising targets for the treatment of cognitive impairment. However, their usefulness can be limited by a number of factors, including addictive properties and adverse side effects [12]. On the one hand, the $\alpha 7$ nicotine acetylcholine receptor (nAChR) subtype seems to mediate the protective effects of nicotine [13-16]. On the other hand, the number of $\beta 4 \alpha 2 \mathrm{nAChRs}$ decreases in $\mathrm{AD}$, while that of $\alpha 7$ remains largely intact, being available for binding to new therapeutic agents [17]. Moreover, $\alpha 7$ $n A C h R s$ do not seem to be related to the addictive effects of nicotine [18]. Preclinical studies in animals, as well as clinical studies in healthy volunteers, have shown procognitive effects of $\alpha 7 \mathrm{nAChRs}[19,20]$. According to the scientific literature, with the exception of nicotine and choline, most $\alpha 7 \mathrm{nAChR}$ agonists have been partial agonists of these receptors [2026]. Little research has been done into selective $\alpha 7 \mathrm{nAChR}$ agonists. Only a few studies on the behavioral effects of this drug have been published. Thus, Bitner and coworkers [11] found that A-582941, a novel $\alpha 7$ nAChR-selective agonist, enhanced the cognitive performance of monkeys, rats, and mice in behavioral studies. In turn, the $\alpha 7 \mathrm{nAChR}$ subtype was specifically shown to be involved in human memory function [10]. Recent investigations suggest that PNU-282987 administered to rats at 1 and $3 \mathrm{mg} / \mathrm{kg}$ can reduce deficiencies in sensory auditory gating induced by amphetamine [27]. This effect seems to be related to an increase in hippocampal GABAergic neurotransmission [28] or to an increase of theta oscillation in the same area [29]. PNU-282987 (PNU) has been shown to be a potent agonist of the $\alpha 7 \mathrm{nAChR}$, having negligible interactions with other nAChR subtypes and being currently the most specific $\alpha 7$ nAChR agonist synthesized [30].

As suggested by Craig and coworkers [31], the neuronal loss or function alteration of cholinergic neurotransmission could lead to a poor ability of neural compensation to cope with secondary insults. It has been shown that different protocols of chronic stress induce alteration in neurotrophic factors [32], oxidative damage, and alterations in antioxidant proteins [33], which may lead to damage in hippocampus. Moreover, the initial hypothesis postulating that the generation of neurons in the postnatal hippocampal dentate gyrus is involved in the etiology and treatment efficacy of major depressive disorders has been also extended for anxiety disorders [34]. Cognitive processes are involved in abnormal early activity reflecting hypervigilance in subcortical networks involving the amygdala and hippocampus [35]. In fact, $\alpha 7$ subunit of the nAChR has been identified as a meaningful intermediary of nicotine's interaction with the stress axis and human disease [36], among others. It has been shown that chronic stress produces changes such as increased $\mathrm{nAChR} \alpha 7 \mathrm{mRNA}$ and decreased receptor binding at hippocampal formation, which suggests that nAChR $\alpha 7$ may be important for the adaptation to stress or hippocampus allostatic load [37].

One of the most interesting plastic processes taking place in the hippocampus is neurogenesis. The concept of neurogenesis was introduced more than 40 years ago [38]. However, it was not until recent decades that a continuous incorporation of new neurons was shown in the adult brain of rodents [39], primates [40], and humans [41]. Moreover, it has been also demonstrated that new generated cells also survive over time being able to establish specific synaptic contacts [42]. Notwithstanding, the functional role of new neurons generated in the adult brain is not well known yet. It has been shown that neurogenesis is induced by exercise and exposure to an enriched environment $[42,43]$, while factors such as advanced age and high levels of glucocorticoids decrease proliferation of new cells [40].

The present study was aimed at evaluating the possible effects of stress in precipitating the onset of deficits in spatial learning and memory in animals with susceptibility to AD. The possible therapeutic roles of PNU $\alpha 7$ agonist as well as the effects of those variables on proliferation of new cells in hippocampus were also investigated.

\section{Materials and Methods}

2.1. Animals and Treatment. Thirty-eight wild type and thirty-nine B6C3-Tg (APPswe, PSEN1De9) 85dB/J transgenic male mice (Charles River, Barcelona, Spain) aged 2 months were used in this study. Animals were quarantined for 10 days after shipping and housed in plastic cages in an animal room, which was maintained at a temperature of $22 \pm 2^{\circ} \mathrm{C}$, a relative humidity of $50 \pm 10 \%$, and on a $12 \mathrm{~h}$ light/dark automatic light cycle (lights on: 08.00 AM-08.00 PM). All animals were allowed free access to food (regular chow diet, Harlan, Barcelona) and tap water. Animals were randomly divided into the following 8 treatment groups.

Wild SAL: $0.9 \%$ saline, not subjected to restraint stress, wild animals $(n=9)$.

Wild SAL-STR: $0.9 \%$ saline, subjected to restraint stress, wild animals $(n=9)$.

Wild PNU: PNU, not subjected to restraint stress, wild animals $(n=10)$.

Wild PNU-STR: PNU, subjected to restraint stress, wild animals $(n=11)$.

Tg SAL: $0.9 \%$ saline, not subjected to restraint stress, transgenic animals $(n=10)$.

Tg SAL-STR: 0.9\% saline, subjected to restraint stress, transgenic animals $(n=9)$. 
Tg PNU: PNU, not subjected to restraint stress, transgenic animals $(n=9)$.

Tg PNU-STR: PNU, subjected to restraint stress, transgenic animals $(n=10)$.

The experimental design of the present study was approved by the Animal Care and Use Committee of the Rovira i Virgili University (Tarragona, Spain), following the "Principles of laboratory animal care." They were carried out in accordance with the European Community Council Directive (86/609/EEC).

2.2. Drugs. The $\alpha 7 \mathrm{nAChR}$ agonist $\mathrm{PNU}$ was purchased from Sigma-Aldrich (Barcelona). The drug was intraperitoneally administered at $1 \mathrm{mg} / \mathrm{kg}$ just before the behavioral tests. The results of a previous study suggested that only at this dose PNU-282987 significantly improved retention in the water maze [44]. PNU was dissolved in $0.9 \%$ saline, the $\mathrm{pH}$ being adjusted to 7. Solutions were administered at volumes of $0.1 \mathrm{~mL} / 10 \mathrm{~g}$ of body weight.

2.3. Restraint Stress. Animals were restrained three times per day (30 min each time) during 30 days. A fixed time gap of $3 \mathrm{~h}$ was established between the restraint sessions. The restraint procedure consisted in placing the mice in metacrylate cylindrical holders from Letica Scientific Instruments (Panlab, Barcelona). Animals were maintained in a prone position. In previous studies, this procedure clearly caused stress in pregnant rodents [45-47].

2.4. Behavioral Tests. To evaluate spatial learning and memory, after a month of restraining animals, were subjected to the Morris water maze test at 3 months of age.

The water maze consisted of a circular tank (diameter $1 \mathrm{~m}$; height $60 \mathrm{~cm}$ ), divided into four quadrants. An escape platform (diameter $10 \mathrm{~cm}$ ) was located $1 \mathrm{~cm}$ below the surface of the water in the target quadrant. Animals performed 4 trials per day for 5 consecutive days. During each trial, mice were allowed $60 \mathrm{~s}$ to find the hidden platform and to remain on it for $30 \mathrm{~s}$. If the animal failed to find the platform within this period, it was placed on it by the experimenter. The order of the three starting positions was randomized throughout the day for each mouse. To avoid proximal cues and prevent egocentric learning, an internal mobile wall was added to the maze, being the wall randomly moved between trials. This seems to increase Morris water maze sensitivity [48]. At the end of the fifth acquisition day, $4 \mathrm{~h}$ after the last training session, retention of the task was assessed by a probe trial, which consisted of a $60 \mathrm{~s}$ free swim without the escape platform. Animal performance was recorded using a video camera placed above the maze. Data were analyzed by the video tracking program EthoVision (Noldus Information Technologies, Wageningen, The Netherlands). Latency to escape the platform, distance traveled, and swimming velocity during the training sessions were measured. During the probe trials, total time spent in the target quadrant and the time spent in other quadrants were also measured in order to compare the time spent searching in the target quadrant with the average time spent in the remaining quadrants.

2.5. Bromodeoxyuridine Administration and Sample Collection. To evaluate hippocampus cell proliferation, two days after behavioral testing, 3 or 4 mice from each group were intraperitoneally injected with 5-bromo-2-deoxyuridine (BrdU) (Sigma, Steinheim, Germany) at $100 \mathrm{mg} / \mathrm{kg} /$ day during two consecutive days [49]. One day after the last BrdU injection, animals were deeply anaesthetized with mixed ketamine-xylazine and sacrificed by decapitation. Brains were rapidly removed from the skulls and divided coronally by free hand in two sections. Two-third posterior brain was postfixed for 4 days at $4^{\circ} \mathrm{C}$ in $4 \%$ paraformaldehyde. On the fifth day, brain samples were transferred to a $30 \%$ sucrose/phosphate buffered solution (PBS) for $48 \mathrm{~h}$ at $4^{\circ} \mathrm{C}$ and then snap-frozen in isopentane. Serial coronal sections, $40 \mu \mathrm{m}$ thick, were cut with a cryostat and collected according to a fractionator principle [50]. Samples were stored at $-20^{\circ} \mathrm{C}$ in a cryoprotection buffer ( $40 \%$ phosphate buffer $0.1 \mathrm{M}, 30 \%$ glycerol, and $30 \%$ ethylene glycol) for later immunohistochemical analyses.

2.6. Immunohistochemistry. One out of six sections was taken for biotinylated-BrdU immunostaining. Free-floating coronal sections of brain were rinsed in TBS and inactivated for endogen phosphatase activity in $0.6 \% \mathrm{H}_{2} \mathrm{O}_{2}$-TBS. Sections were then treated for DNA denaturation incubating in $2 \mathrm{M}$ $\mathrm{HCl}$ at $37^{\circ} \mathrm{C}$ and rinsed in $0.1 \mathrm{M}$ sodium borate buffer. Sections were blocked in TBS-Plus containing 3\% normal goat serum and $1 \%$ Triton-X in TBS for $30 \mathrm{~min}$. Antibody against BrdU (Serotec, Oxford, UK) was diluted 1:500 in blocking buffer and incubated overnight at $4^{\circ} \mathrm{C}$. Following this incubation, tissue sections were washed with TBS-Plus and incubated with secondary antibody (biotinylated anti-rat IgG, 1:500, Vector Laboratories, Burlingame, USA) for $2 \mathrm{~h}$. After additional washes, the secondary antibody was detected using the avidin-biotin complex reaction (ABC Elite Kit). Diaminobenzidine (Vector Laboratories, Burlingame, USA) was used as chromogen. Sections were thoroughly washed, mounted, and cover-slipped.

2.7. Quantification of BrdU Positive Cells. Data for proliferation were obtained by using methods of unbiased stereology. The hippocampus was identified by anatomical criteria following the Mouse Brain Library Atlas [51] available at http://www.mbl.org/. The total numbers of BrdU positive cells in the granular cell layer and hilus of the bilateral entire hippocampus were exhaustively counted in serial coronal brain sections. Each section was $240 \mu \mathrm{m}$ apart from each other. The set of selected sections represents one-sixth of the whole hippocampus, being representative of the total hippocampus. Positive cells were counted by means of an optic microscopy (Olympus, $\mathrm{CH} 20$ ) through a 100× objective. Cells in the uppermost focal plane were discarded to avoid counting twice cells cut in two parts [52]. As usually, to yield an estimate of the number of BrdU positive cell numbers in the entire structure, the numbers counted were multiplied by 6 [53]. 
TABLE 1: Total number of BrdU positive cells in wild type and B6C3-Tg mice.

\begin{tabular}{lcccr}
\hline & SAL & SAL/STR & PNU & PNU/STR \\
\hline Wild type mice & $177.0 \pm 53.81$ & $125.3 \pm 26.13$ & $413.7 \pm 122.67$ & $286.8 \pm 96.32$ \\
B6C3-Tg mice & $115.7 \pm 25.74$ & $215.0 \pm 22.30$ & $295.0 \pm 30.40$ & $115.7 \pm 12.30$ \\
\hline
\end{tabular}

Data are expressed as group means \pm SEM.

2.8. Statistics. Behavioral data were analyzed by means of a three-way ANOVA (Genotype $\times$ Stress $\times$ Drug). Post hoc Tukey test was used to analyze the differences between groups. Analyses for variance homogeneity were performed by means of Levene's test. A Welch's $F$ and Post hoc Dunnett's T3 test were used when appropriated. One-way ANOVA for repeated measures was used to analyze spatial learning during the acquisition of the water maze. To analyze the differences between groups, a Bonferroni-adjusted pairwise comparison was used when necessary. Student's $t$-test was used for searching differences between transgenic mice and their respective wild type control groups. Nonparametrical analysis for cell proliferation was used. Statistical significance was set at $P<0.05$.

\section{Results}

ANOVA for repeated measures indicated an overall effect on Group $(F(7,68)=6.162 ; P<0.001)$, Day $(F(4,65)=$ $70.509 ; P<0.001)$, Day $\times$ Group $(F(28,272)=2.776 ; P<$ $0.001)$, and Day $\times$ Genotype $(F(4,65)=15.383 ; P<$ $0.001)$ during acquisition of the water maze. Post hoc Tukey test showed that the latency to escape the platform was significantly higher in stressed and nonstressed transgenic mice $(P=0.038$ and $P=0.015)$, in comparison to the corresponding wild mice groups (Figure 1(a)). These differences were noted on days $3(P<0.001), 4(P=0.013)$, and $5(P=0.044)$ on stressed animals but only on days $3(P<$ $0.001)$ and $4(P=0.013)$ in control animals (Figure $1(\mathrm{~b}))$. There was no significant effect of chronic stress on latency to escape the platform, in both wild type and transgenic mice. Moreover, there was no significant effect of drug treatment on the latency to escape the platform in any group. No significant differences in the distance traveled through the acquisition days were found (data not shown).

Retention of the water maze was evaluated by a probe trial performed $4 \mathrm{~h}$ after the last acquisition trial. Results showed an interaction effect between Genotype $\times$ Stress $\times$ $\operatorname{Drug}(F(3,13)=4.679 ; P=0.020)$. A significant difference between wild and transgenic mice receiving $\mathrm{PNU}(t=2.088$, $f d=36, P=0.044$ ) was observed on the time spent in the target quadrant (Figure 2).

In order to better analyze differences on retention, we compared time spent in the target quadrant with the mean time spent in the other three quadrants. Differences emerged on stressed wild animals ( $t=2.678, f d=9, P=0.025)$, PNU wild group ( $t=3.269, f d=8, P=0.011)$, and stressed wild mice given PNU $(t=5.782, f d=8, P<0.001)$ (Figure 3 ).

No significant differences were detected on new cell proliferation (Table 1), while no correlations were noted between the number of BdrU positive cells and performance in the probe trial.

\section{Discussion}

Since cholinergic neurodegeneration has been related to cognitive deterioration in $\mathrm{AD}$ [11], agents enhancing nicotinic cholinergic transmission have been identified as promising targets for the treatment of cognitive impairment [54]. It has been demonstrated that chronic stress accelerates cognitive decline and increases extracellular deposits of $\mathrm{A} \beta$ protein in a transgenic model of $\mathrm{AD}$ [9]. The aim of the present investigation was to evaluate in a transgenic model of $\mathrm{AD}$ the possible precipitating effects of stress in the onset of cognitive deterioration in AD. The therapeutic role of PNU-282987, an $\alpha 7$ cholinergic agonist, was evaluated. The effects of stress and PNU on the proliferation of new cells in hippocampus of animals with susceptibility to AD were also assessed.

The results indicated that although all animals learned the water maze, differences in the learning curve were noted. Although transgenic mice acquired more slowly the task, they reached similar levels as wild mice. Significant differences only emerged at the end of the learning period between wild and transgenic stressed animals, indicating a differential effect of stress depending on the genetic characteristics of the mice. It is important to consider that the current results showed a deficit in learning process in transgenic control mice compared to wild animals given $0.9 \%$ saline only (Figure 1(a)). Stress condition did not show any effect on acquisition in both wild and transgenic mice. Our data showed a possible difference in the learning basal line between transgenic and wild mice, which is probably related to the genetic characteristics of the groups. However, it is important to note that this difference was eliminated by PNU administration. No differences between animals with different $\mathrm{AD}$ vulnerability receiving PNU, with or without stress, were detected. These results suggest that PNU is able to improve acquisition in transgenic mice. In relation to this, it has been shown that PNU could reverse spatial deficits evaluated by a 12 -arm radial maze test in rats at $3 \mathrm{mg} / \mathrm{kg}$ [55]. Moreover, although no effect on acquisition was detected in C57BL/6J, a better retention was noted after administration of $1 \mathrm{mg} / \mathrm{kg}$ of PNU [44].

With regard to spatial memory, no significant differences were observed between any experimental condition, neither in wild mice, nor in transgenic mice. Notwithstanding, a significant difference was detected between wild and transgenic mice receiving PNU. In order to better understand this difference, another analysis was performed to compare the time spent in the target quadrant with the mean time spent 


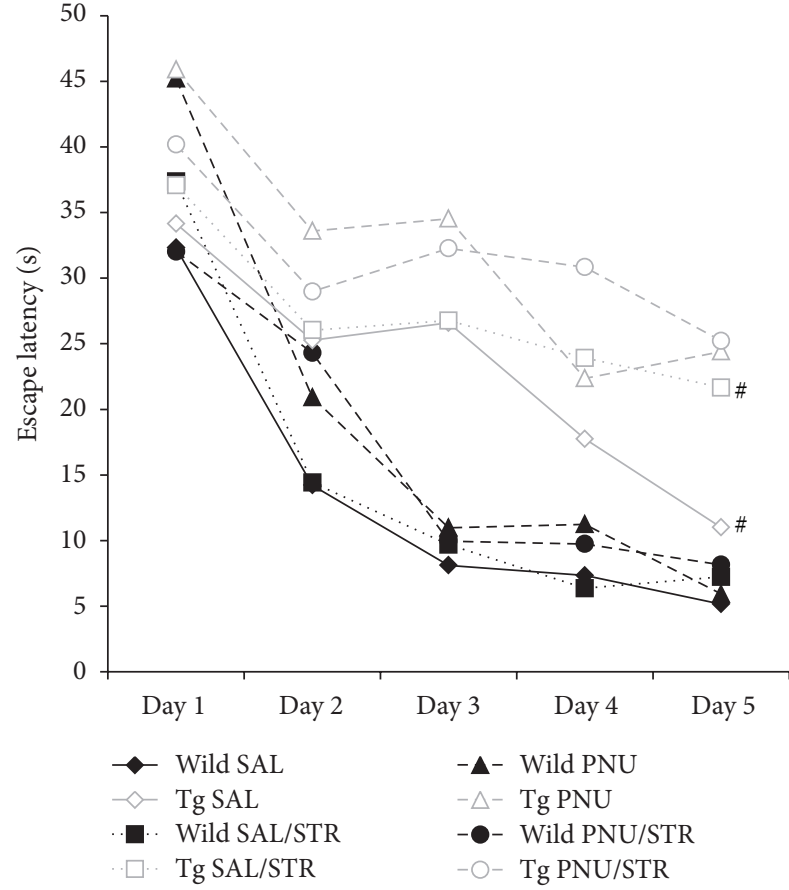

(a)

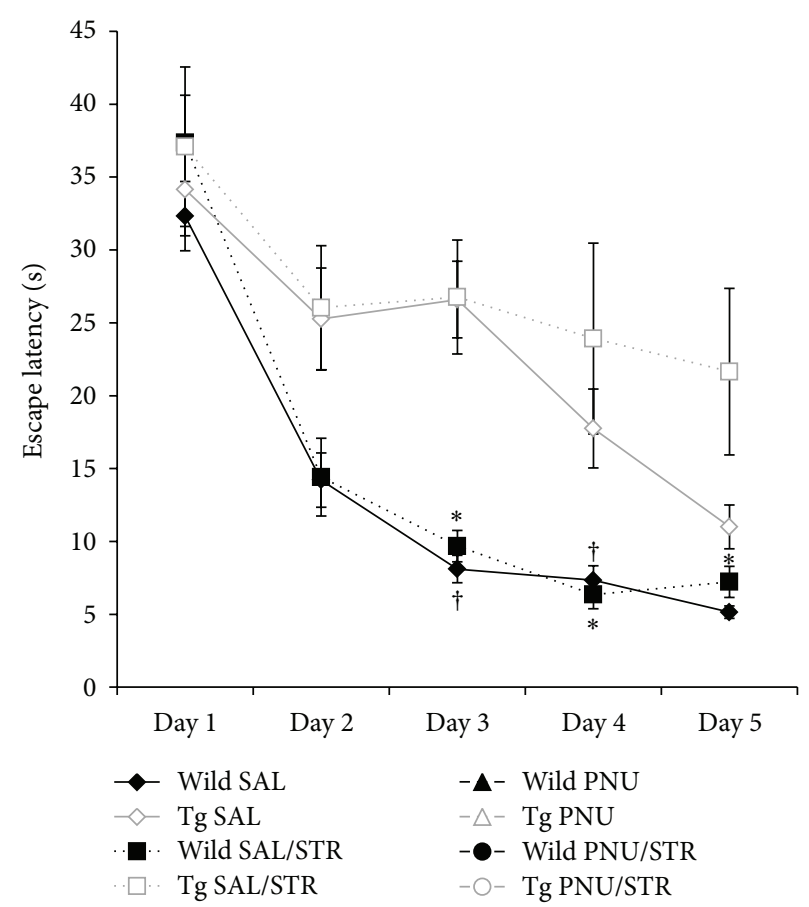

(b)

Figure 1: Acquisition of the Morris water maze. (a) Escape latencies in each group. A hash symbol indicates significant differences compared to their corresponding wild type groups at $P<0.05$. (b) In order to better show differences between groups, (b) only shows escape latencies in wild type and B6C3-Tg 0.9\% saline groups. Data are expressed as mean values \pm SEM. An asterisk indicates significant differences between stressed wild and stressed transgenic mice at $P<0.05$. A cross indicates significant differences between wild and transgenic saline groups.

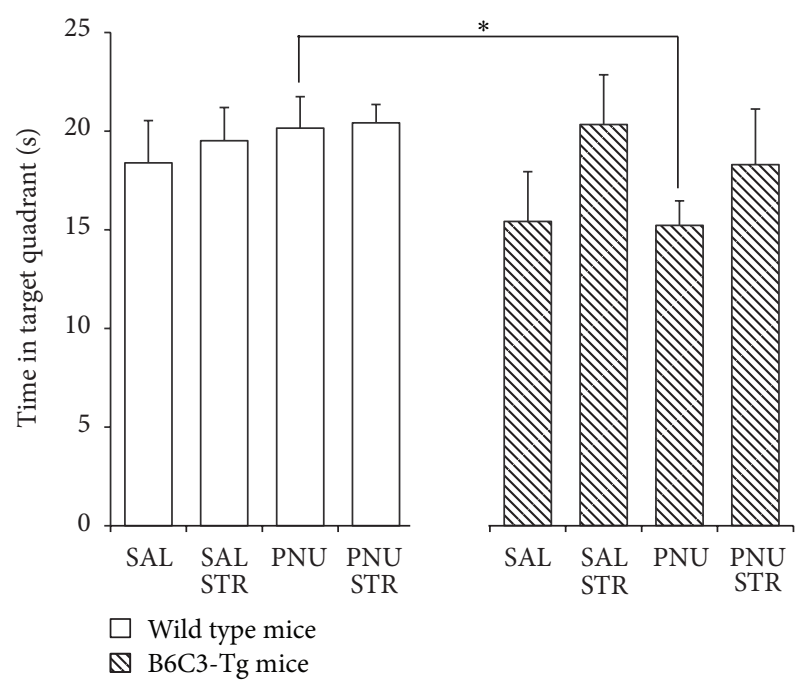

FIGURE 2: Time spent in the target quadrant $4 \mathrm{~h}$ after the last training session for wild type and B6C3-Tg mice. Data are expressed as mean values \pm SEM. An asterisk indicates significant differences between groups at $P<0.05$.

in the other three quadrants $[49,56]$. Stress condition only, as well as PNU administration, with or without immobilization stress, improved retention of the task in wild mice only. PNU and stress had significant effects on memory on wild

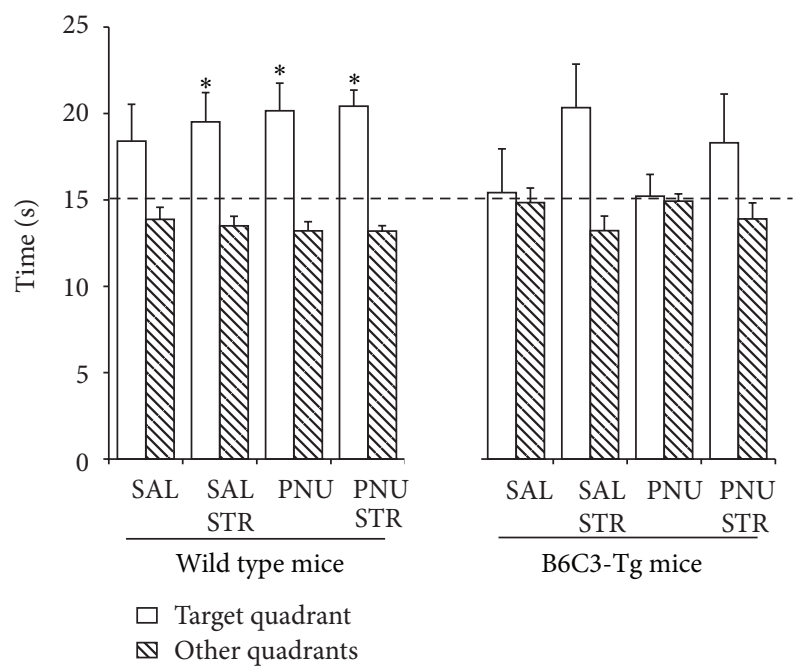

FIgURE 3: Time spent in the target quadrant in relation to other quadrants $4 \mathrm{~h}$ after the last training session for wild type and B6C3Tg mice. Data are expressed as mean values \pm SEM. An asterisk indicates significant differences at $P<0.05$.

type animals improving their performance. However, the results did not show additive effects. Previous studies have demonstrated that moderate stress could enhance spatial memory tasks in rats [57] and mice [58]. 
No significant differences in new cell proliferation were found, which indicated no effects of stress on this neurogenesis process. In the scientific literature, multiple protocols to induce stress to mice may be found [59]. However, there are only few available studies using restrained stress in transgenic mice. Regarding this, in a recent study, Tg (APPswe/PS1dE) mice were subjected to $2 \mathrm{~h}$ per day of restraint stress during 16 consecutive days ( $32 \mathrm{~h}$ in total), showing oxidative stress increase and mitochondrial dysfunction [60]. Another recent investigation demonstrated that $6 \mathrm{~h} /$ day of restraint for 3 weeks excluding weekends ( $90 \mathrm{~h}$ in total) and also for 15 weeks $(450 \mathrm{~h})$ could efficiently induce typical physical stress symptoms in a transgenic mice model and increase hippocampal neurodegeneration [61]. In the present study, a similar number of hours were used, noting similar effects. However, this must be corroborated in further investigations, because no effects on neurogenesis were detected, while in contrast, other authors have shown clear effects of stress on new cell proliferation [62]. Moreover, we failed to find PNU or genotype effects on new cell proliferation. This could be related to the fact that transgenic mice were too young, and cell proliferation was not reduced by age, as usual. In fact, these animals have similar proliferation levels as wild type mice. It has been reported that although stress could disrupt spatial working memory both in adult an aged mice, aged animals are more sensitive [63].

In summary, the current data suggest possible effects of PNU on acquisition of a spatial task in transgenic mice, which should be more accurately evaluated. In this sense, it should be interesting to test the effects of an $\alpha 7 \mathrm{nAChR}$ antagonist. In turn, PNU and stress effects were detected on retention of the task in wild type animals, but no changes were detected in transgenic mice. To better understand interactions between environmental and genetic factors in $\mathrm{AD}$, further studies are clearly necessary. According to the results of the present study, a longer PNU administration and/or older animals could be used for determining whether stress and PNU effects are modified by age.

\section{Acknowledgment}

This research was supported by the Spanish Ministry of Science though Grant SEJ2007-63369.

\section{References}

[1] R. A. Fuentealba, G. Farias, J. Scheu, M. Bronfman, M. P. Marzolo, and N. C. Inestrosa, "Signal transduction during amyloid- $\beta$-peptide neurotoxicity: role in Alzheimer disease," Brain Research Reviews, vol. 47, no. 1-3, pp. 275-289, 2004.

[2] L. M. Bekris, C.-E. Yu, T. D. Bird, and D. W. Tsuang, "Review article: genetics of Alzheimer disease," Journal of Geriatric Psychiatry and Neurology, vol. 23, no. 4, pp. 213-227, 2010.

[3] E. L. Schaeffer, M. Figueiró, and W. F. Gattaz, "Insights into Alzheimer disease pathogenesis from studies in transgenic animal models," Clinics, vol. 66, no. 1, pp. 45-54, 2011.

[4] J. L. Jankowsky, H. H. Slunt, T. Ratovitski, N. A. Jenkins, N. G. Copeland, and D. R. Borchelt, "Co-expression of multiple transgenes in mouse CNS: a comparison of strategies," Biomolecular Engineering, vol. 17, no. 6, pp. 157-165, 2001.
[5] R. S. Reiserer, F. E. Harrison, D. C. Syverud, and M. P. McDonald, "Impaired spatial learning in the APPSwe + PSEN1 $\triangle \mathrm{E} 9$ bigenic mouse model of Alzheimer's disease," Genes, Brain and Behavior, vol. 6, no. 1, pp. 54-65, 2007.

[6] A. Savonenko, G. M. Xu, T. Melnikova et al., "Episodiclike memory deficits in the APPswe/PS1dE9 mouse model of Alzheimer's disease: relationships to $\beta$-amyloid deposition and neurotransmitter abnormalities," Neurobiology of Disease, vol. 18, no. 3, pp. 602-617, 2005.

[7] B. S. McEwen, "The neurobiology of stress: from serendipity to clinical relevance," Brain Research, vol. 886, no. 1-2, pp. 172-189, 2000.

[8] B. S. McEwen, "Protective and damaging effects of stress mediators: the good and bad sides of the response to stress," Metabolism, vol. 51, no. 6, pp. 2-4, 2002.

[9] H. J. Yun, H. P. Cheol, J. Yoo et al., "Chronic stress accelerates learning and memory impairments and increases amyloid deposition in APPV717I-CT100 transgenic mice, an Alzheimer's disease model," FASEB Journal, vol. 20, no. 6, pp. 729-731, 2006.

[10] K. Mizoguchi, M. Yuzurihara, A. Ishige, H. Sasaki, and T. Tabira, "Effect of chronic stress on cholinergic transmission in rat hippocampus," Brain Research, vol. 915, no. 1, pp. 108-111, 2001.

[11] R. S. Bitner, W. H. Bunnelle, D. J. Anderson et al., "Broadspectrum efficacy across cognitive domains by $\alpha 7$ nicotinic acetylcholine receptor agonism correlates with activation of ERK1/2 and CREB phosphorylation pathways," Journal of Neuroscience, vol. 27, no. 39, pp. 10578-10587, 2007.

[12] V. Riljak and M. Langmeier, "Nicotine an efficient tool of the neurobiological research today, the tool of treatment tomorrow?" Prague Medical Report, vol. 106, no. 4, pp. 329-348, 2005.

[13] M. R. D’Andrea and R. G. Nagele, “Targeting the alpha 7 nicotinic acetylcholine receptor to reduce amyloid accumulation in Alzheimer's disease pyramidal neurons," Current Pharmaceutical Design, vol. 12, no. 6, pp. 677-684, 2006.

[14] Q. Liu and B. Zhao, "Nicotine attenuates $\beta$-amyloid peptideindueed neurotoxicity, free radical and calcium accumulation in hippocampal neuronal cultures," British Journal of Pharmacology, vol. 141, no. 4, pp. 746-754, 2004.

[15] A. Mazurov, T. Hauser, and C. H. Miller, "Selective $\alpha 7$ nicotinic acetylcholine receptor ligands," Current Medicinal Chemistry, vol. 13, no. 13, pp. 1567-1584, 2006.

[16] X. D. Li and J. J. Buccafusco, "Role of $\alpha 7$ nicotinic acetylcholine receptors in the pressor response to intracerebroventricular injection of choline: blockade by amyloid peptide A $\beta 1-42$," Journal of Pharmacology and Experimental Therapeutics, vol. 309, no. 3, pp. 1206-1212, 2004.

[17] W. R. Kem, "The brain $\alpha 7$ nicotinic receptor may be an important therapeutic target for the treatment of Alzheimer's disease: studies with DMXBA (GTS-21)," Behavioural Brain Research, vol. 113, no. 1-2, pp. 169-181, 2000.

[18] J. W. Young, N. Crawford, J. S. Kelly et al., "Impaired attention is central to the cognitive deficits observed in alpha 7 deficient mice," European Neuropsychopharmacology, vol. 17, no. 2, pp. 145-155, 2007.

[19] H. Kitagawa, T. Takenouchi, R. Azuma et al., "Safety, pharmacokinetics, and effects on cognitive function of multiple doses of GTS-21 in healthy, male volunteers," Neuropsychopharmacology, vol. 28, no. 3, pp. 542-551, 2003.

[20] J. J. Buccafusco, S. R. Letchworth, M. Bencherif, and P. M. Lippiello, "Long-lasting cognitive improvement with nicotinic 
receptor agonists: mechanisms of pharmacokinetic-pharmacodynamic discordance," Trends in Pharmacological Sciences, vol. 26, no. 7, pp. 352-360, 2005.

[21] M. Nanri, H. Miyake, Y. Murakami, K. Matsumoto, and H. Watanabe, "GTS-21, a nicotinic agonist, attenuates multiple infarctions and cognitive deficit caused by permanent occlusion of bilateral common carotid arteries in rats," Japanese Journal of Pharmacology, vol. 78, no. 4, pp. 463-469, 1998.

[22] M. Nanri, N. Kasahara, J. Yamamoto, H. Miyake, and H. Watanabe, "A comparative study on the effects of nicotine and GTS-21, a new nicotinic agonist, on the locomotor activity and brain monoamine level," Japanese Journal of Pharmacology, vol. 78, no. 3, pp. 385-389, 1998.

[23] M. Nanri, N. Kasahara, J. Yamamoto, H. Miyake, and H. Watanabe, "GTS-21, a nicotinic agonist, protects against neocortical neuronal cell loss induced by the nucleus basalis magnocellularis lesion in rats," Japanese Journal of Pharmacology, vol. 74, no. 3, pp. 285-289, 1997.

[24] A. Olincy and K. E. Stevens, "Treating schizophrenia symptoms with an $\alpha 7$ nicotinic agonist, from mice to men," Biochemical Pharmacology, vol. 74, no. 8, pp. 1192-1201, 2007.

[25] L. Lagostena, C. Trocme-Thibierge, P. Morain, and E. Cherubini, "The partial $\alpha 7$ nicotine acetylcholine receptor agonist S 24795 enhances long-term potentiation at CA3-CA1 synapses in the adult mouse hippocampus," Neuropharmacology, vol. 54, no. 4, pp. 676-685, 2008.

[26] M. S. Thomsen, D. Z. Christensen, H. H. Hansen, J. P. Redrobe, and J. D. Mikkelsen, " $\alpha 7$ Nicotinic acetylcholine receptor activation prevents behavioral and molecular changes induced by repeated phencyclidine treatment," Neuropharmacology, vol. 56, no. 6-7, pp. 1001-1009, 2009.

[27] A. L. Bodnar, L. A. Cortes-Burgos, K. K. Cook et al., "Discovery and structure-activity relationship of quinuclidine benzamides as agonists of $\alpha 7$ nicotinic acetylcholine receptors," Journal of Medicinal Chemistry, vol. 48, no. 4, pp. 905-908, 2005.

[28] M. Hajós, R. S. Hurst, W. E. Hoffmann et al., "The selective $\alpha 7$ nicotinic acetylcholine receptor agonist PNU-282987 [N[(3R)-1-azabicyclo[2.2.2] oct-3-yl]-4-chlorobenzamide hydrochloride] enhances GABAergic synaptic activity in brain slices and restores auditory gating deficits in anesthetized rats," Journal of Pharmacology and Experimental Therapeutics, vol. 312, no. 3, pp. 1213-1222, 2005.

[29] C. J. Siok, J. A. Rogers, B. Kocsis, and M. Hajós, "Activation of $\alpha 7$ acetylcholine receptors augments stimulation-induced hippocampal theta oscillation," European Journal of Neuroscience, vol. 23, no. 2, pp. 570-574, 2006.

[30] W. K. Chan, P. Tsun-Hon Wong, and F.-S. Sheu, "Frontal cortical $\alpha 7$ and $\alpha 4 \beta 2$ nicotinic acetylcholine receptors in working and reference memory," Neuropharmacology, vol. 52, no. 8, pp. 16411649, 2007.

[31] L. A. Craig, N. S. Hong, and R. J. McDonald, "Revisiting the cholinergic hypothesis in the development of Alzheimer's disease," Neuroscience and Biobehavioral Reviews, vol. 35, no. 6, pp. 1397-1409, 2011.

[32] S.-H. Shao, S.-S. Shi, Z.-L. Li, M.-S. Zhao, S.-Y. Xie, and F. Pan, "Aging effects on the BDNF mRNA and TrkB mRNA expression of the hippocampus in different durations of stress," Chinese Journal of Physiology, vol. 53, no. 5, pp. 285-293, 2010.

[33] C. Wang, H. M. Wu, X. R. Jing et al., "Oxidative parameters in the rat brain of chronic mild stress model for depression: relation to anhedonia-like responses," The Journal of Membrane Biology, vol. 245, no. 11, pp. 675-681, 2012.
[34] D. Petrik, D. C. Lagace, and A. J. Eisch, “The neurogenesis hypothesis of affective and anxiety disorders: are we mistaking the scaffolding for the building?" Neuropharmacology, vol. 62, no. 1, pp. 21-34, 2012.

[35] S. G. Hofmann, K. K. Ellard, and G. J. Siegle, "Neurobiological correlates of cognitions in fear and anxiety: a cognitiveneurobiological information-processing model," Cognition and Emotion, vol. 26, no. 2, pp. 282-299, 2012.

[36] R. G. Hunter, "Stress and the $\alpha 7$ nicotinic acetylcholine receptor," Current Drug Targets, vol. 13, no. 5, pp. 607-612, 2012.

[37] R. G. Hunter, E. B. Bloss, K. J. McCarthy, and B. S. McEwen, "Regulation of the nicotinic receptor alpha7 subunit by chronic stress and corticosteroids," Brain Research, vol. 1325, pp. 141-146, 2010.

[38] J. Altman and G. D. Das, "Autoradiographic and histological evidence of postnatal hippocampal neurogenesis in rats," Journal of Comparative Neurology, vol. 124, no. 3, pp. 319-335, 1965.

[39] M. S. Kaplan and J. W. Hinds, "Neurogenesis in the adult rat: electron microscopic analysis of light radioautographs," Science, vol. 197, no. 4308, pp. 1092-1094, 1977.

[40] E. Gould, B. S. McEwen, P. Tanapat, L. A. M. Galea, and E. Fuchs, "Neurogenesis in the dentate gyrus of the adult tree shrew is regulated by psychosocial stress and NMDA receptor activation," Journal of Neuroscience, vol. 17, no. 7, pp. 2492-2498, 1997.

[41] P. S. Eriksson, E. Perfilieva, T. Björk-Eriksson et al., "Neurogenesis in the adult human hippocampus," Nature Medicine, vol. 4, no. 11, pp. 1313-1317, 1998.

[42] H. Van Praag, A. F. Schinder, B. R. Christle, N. Toni, T. D. Palmer, and F. H. Gage, "Functional neurogenesis in the adult hippocampus," Nature, vol. 415, no. 6875, pp. 1030-1034, 2002.

[43] G. Kempermann, H. G. Kuhn, and F. H. Gage, "More hippocampal neurons in adult mice living in an enriched environment," Nature, vol. 386, no. 6624, pp. 493-495, 1997.

[44] P. Vicens, D. Ribes, M. Torrente, and J. L. Domingo, "Behavioral effects of PNU-282987, an alpha7 nicotinic receptor agonist, in mice," Behavioural Brain Research, vol. 216, no. 1, pp. 341-348, 2011.

[45] M. L. Albina, M. Bellés, V. Linares, D. J. Sánchez, and J. L. Domingo, "Restraint stress does not enhance the uraniuminduced developmental and behavioral effects in the offspring of uranium-exposed male rats," Toxicology, vol. 215, no. 1-2, pp. 69-79, 2005.

[46] M. T. Colomina, J. L. Roig, M. Torrente, P. Vicens, and J. L. Domingo, "Concurrent exposure to aluminum and stress during pregnancy in rats: effects on postnatal development and behavior of the offspring," Neurotoxicology and Teratology, vol. 27, no. 4, pp. 565-574, 2005.

[47] S. Fuentes, M. T. Colomina, J. Rodriguez, P. Vicens, and J. L. Domingo, "Interactions in developmental toxicology: concurrent exposure to perfluorooctane sulfonate (PFOS) and stress in pregnant mice," Toxicology Letters, vol. 164, no. 1, pp. 81-89, 2006.

[48] D. Ribes, M. T. Colomina, P. Vicens, and J. L. Domingo, "Effects of oral aluminum exposure on behavior and neurogenesis in a transgenic mouse model of Alzheimer's disease," Experimental Neurology, vol. 214, no. 2, pp. 293-300, 2008.

[49] D. Ribes, M. T. Colomina, P. Vicens, and J. L. Domingo, "Impaired spatial learning and unaltered neurogenesis in a transgenic model of alzheimer's disease after oral aluminum exposure," Current Alzheimer Research, vol. 7, no. 5, pp. 401-408, 2010. 
[50] M. J. West, L. Slomianka, and H. J. G. Gundersen, "Unbiased stereological estimation of the total number of neurons in the subdivisions of the rat hippocampus using the optical fractionator," Anatomical Record, vol. 231, no. 4, pp. 482-497, 1991.

[51] G. Rosen et al., "The mouse brain library," in 2000, vol. 14, p. 166, International Mouse Genome Conference, http:// www.mbl.org/.

[52] J. M. Encinas and G. Enikolopov, "Identifying and quantitating neural stem and progenitor cells in the adult brain," Methods in Cell Biology, vol. 85, pp. 243-272, 2008.

[53] D. Ehninger, L.-P. Wang, F. Klempin, B. Römer, H. Kettenmann, and G. Kempermann, "Enriched environment and physical activity reduce microglia and influence the fate of NG2 cells in the amygdala of adult mice," Cell and Tissue Research, vol. 345, no. 1, pp. 69-86, 2011.

[54] R. W. Gould, P. K. Garg, S. Garg, and M. A. Nader, "Effects of nicotinic acetylcholine receptor agonists on cognition in rhesus monkeys with a chronic cocaine self-administration history," Neuropharmacology, vol. 64, pp. 479-488, 2013.

[55] A. A. Pandya and J. L. Yakel, "Activation of the alpha7 nicotinic ACh receptor induces anxiogenic effects in rats which is blocked by a 5-HT receptor antagonist," Neuropharmacology, vol. 70C, pp. 35-42, 2013.

[56] L. Heredia, M. Torrente, M. T. Colomina, and J. L. Domingo, "Behavioral effects of oral subacute exposure to BDE-209 in young adult mice: a preliminary study," Food and Chemical Toxicology, vol. 50, no. 3-4, pp. 707-712, 2012.

[57] I. Akirav, M. Kozenicky, D. Tal, C. Sandi, C. Venero, and G. Richter-Levin, "A Facilitative Role for Corticosterone in the Acquisition of a Spatial Task under Moderate Stress," Learning and Memory, vol. 11, no. 2, pp. 188-195, 2004.

[58] E. Galliot, M. Levaillant, E. Beard, J.-L. Millot, and G. Pourié, "Enhancement of spatial learning by predator odor in mice: involvement of amygdala and hippocampus," Neurobiology of Learning and Memory, vol. 93, no. 2, pp. 196-202, 2010.

[59] C. D. Conrad, "A critical review of chronic stress effects on spatial learning and memory," Progress in Neuro-Psychopharmacology and Biological Psychiatry, vol. 34, no. 5, pp. 742-755, 2010.

[60] J.-S. Seo, K.-W. Lee, T.-K. Kim, I.-S. Baek, J.-Y. Im, and P.-L. Han, "Behavioral stress causes mitochondrial dysfunction via $\mathrm{ABAD}$ up-regulation and aggravates plaque pathology in the brain of a mouse model of Alzheimer disease," Free Radical Biology and Medicine, vol. 50, no. 11, pp. 1526-1535, 2011.

[61] S. Kunimoto, S. Nakamura, K. Wada, and T. Inoue, "Chronic stress-mutated presenilin 1 gene interaction perturbs neurogenesis and accelerates neurodegeneration," Experimental Neurology, vol. 221, no. 1, pp. 175-185, 2010.

[62] J. L. Pawluski, D. L. A. van den Hove, I. Rayen, J. Prickaerts, and H. W. M. Steinbusch, "Stress and the pregnant female: impact on hippocampal cell proliferation, but not affective-like behaviors," Hormones and Behavior, vol. 59, no. 4, pp. 572-580, 2011.

[63] J. B. Buchanan, N. L. Sparkman, J. Chen, and R. W. Johnson, "Cognitive and neuroinflammatory consequences of mild repeated stress are exacerbated in aged mice," Psychoneuroendocrinology, vol. 33, no. 6, pp. 755-765, 2008. 

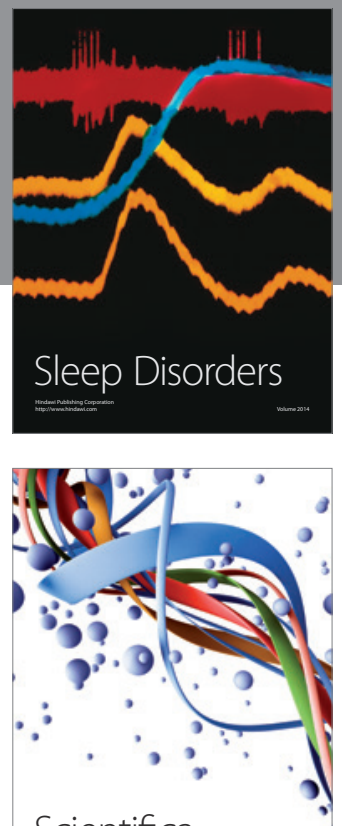

Scientifica
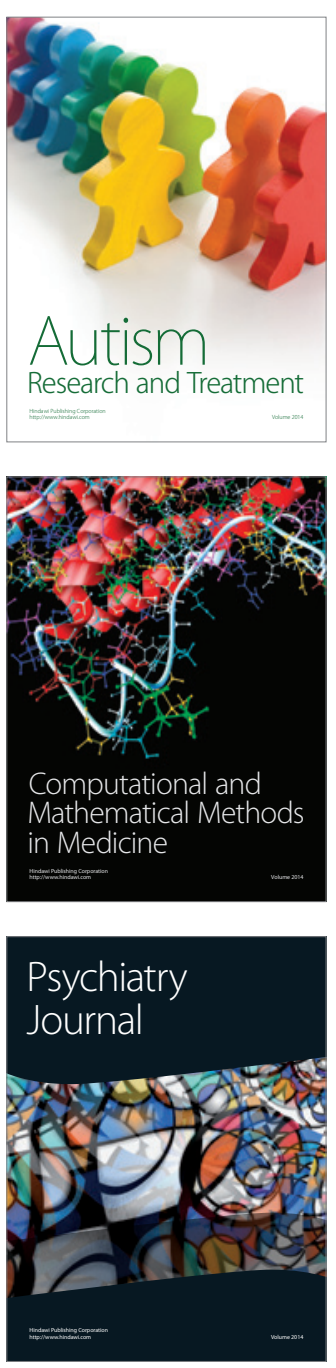
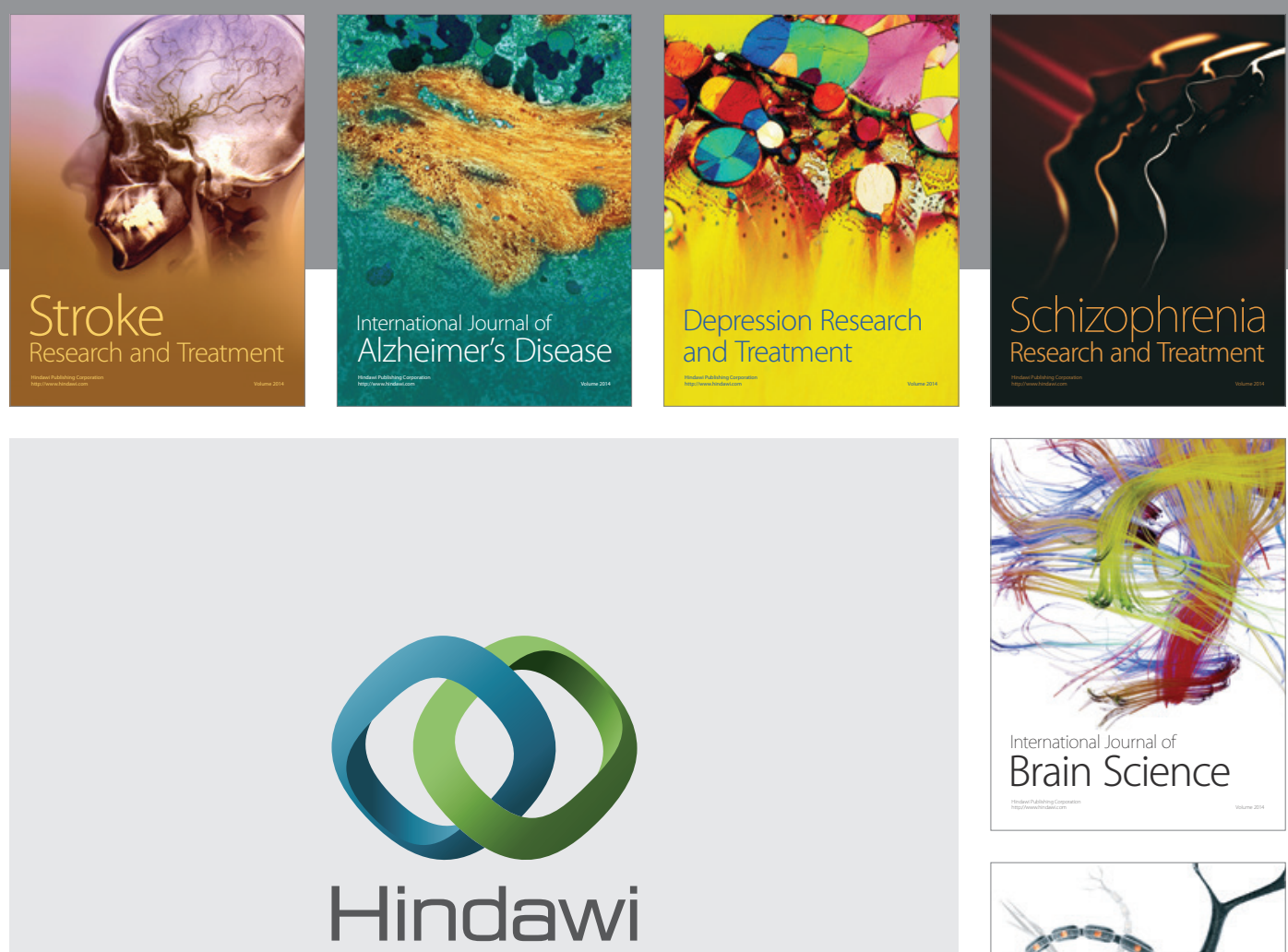

Submit your manuscripts at

http://www.hindawi.com
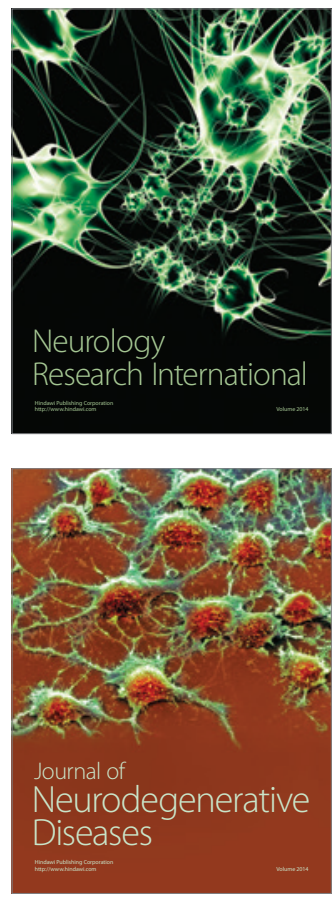

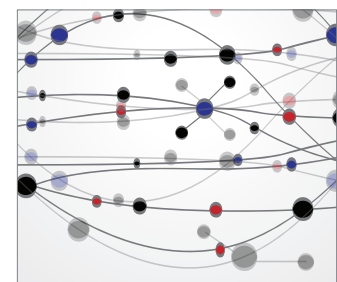

The Scientific World Journal
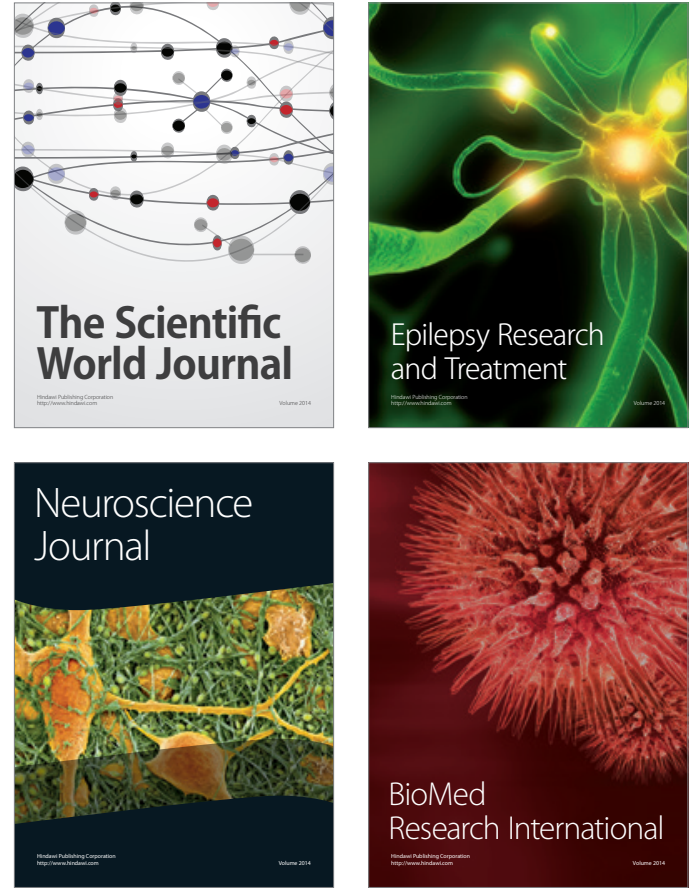

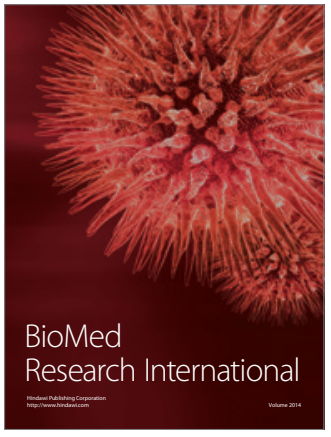

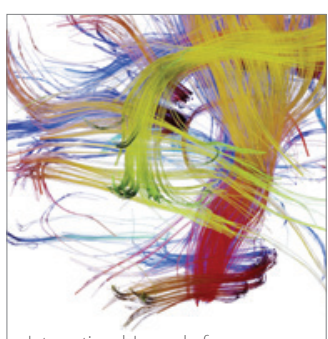

Brain Science

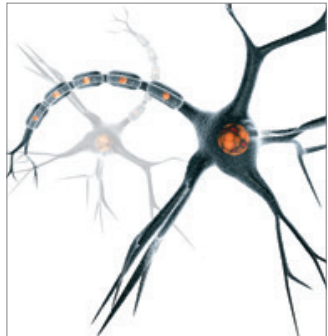

Neural Plasticity
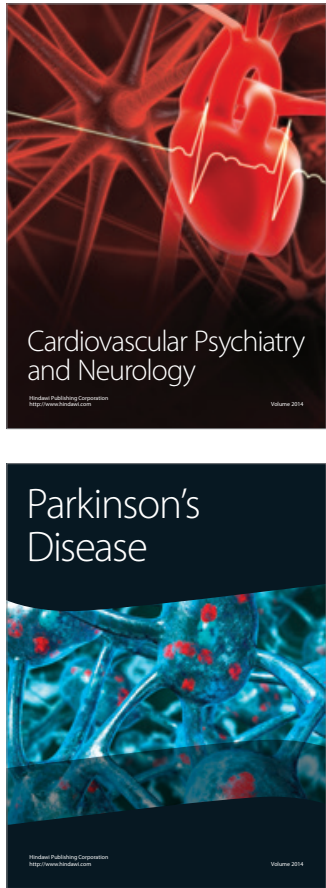